Noname manuscript No.

(will be inserted by the editor)

\title{
Coupling Fishery Dynamics, Human Health and Social Learning in a Model of Fish-borne Pollution Exposure
}

\author{
Michael Yodzis • Chris T. Bauch • \\ Madhur Anand
}

Received: date / Accepted: date

\begin{abstract}
Pollution-induced illnesses are caused by toxicants that result from human activity and are often entirely preventable. However, where industrial priorities have undermined responsible governance, exposed populations must reduce their exposure by resorting to voluntary protective measures and demanding emissions abatement. This paper presents a coupled humanenvironment system (HES) model that represents the effects of water pollution on the health and livelihood of a fishing community. The model is motivated by an incident from 1949-1968 in Minamata, Japan, where methylmercury effluent from a local factory poisoned fish populations and humans who ate them. We model the critical role of risk perception in driving both social learning and the protective feedbacks against pollution exposure. These feedbacks are undermined in the presence of social misperceptions such as stigmatization of the injured. Through numerical simulation and scenario analysis, we compare our model results with historical datasets from Minamata, and find that the conditions for an ongoing pollution epidemic are highly unlikely without social misperception. We also find trade-offs between human health outcomes, the viability of the polluting industry and the survival of the fishery. We conclude that an understanding of human-environment interactions and misperception
\end{abstract}

\author{
OGS, NSERC \\ Michael Yodzis \\ Department of Mathematics, University of Guelph, 50 Stone Road E., \\ Guelph, ON N1G 2W1, Canada \\ Tel.: +1-519-824-4120 ext.52155 \\ Fax: +1-519-837-0221 \\ E-mail: myodzis@uoguelph.ca \\ Chris T. Bauch \\ Department of Applied Mathematics, University of Waterloo \\ Madhur Anand \\ School of Environmental Sciences, University of Guelph
}


effects is highly relevant to the resolution of contemporary pollution problems, and merits further study.

Keywords human-environment interactions, social learning, pollution abatement, misperception effects, trade-offs

\section{Introduction}

A human-environment system (HES) is characterized by the interaction of human activities and natural processes (Liu et al 2007; Levin and Clark 2010), and may also be called a socio-ecological system (SES) when biota are involved (Anderies 2014). Pollution effects on populations and natural resources and the human responses to those effects can exemplify human-environment interactions: human activities can cause an increase in pollution, which in turn can feed back to change human perception and behaviour, and possibly stimulate efforts to control the pollution.

For some applications, mathematical modellers assume that a policymaker wishes to ameliorate environmental impacts, and has the power to make a decision under constraints. Under these conditions, it is possible to use optimal control theory to understand how the policymaker can optimize the amelioration efforts. This approach is used in the context of pollution HESs and similar systems involving humans and their environment (Withagen 1995; Eichner and Pethig 2006; Dubey 2010; Heijnen and Wagener 2013; Anderies 2014). However, for other applications, it may be desirable to incorporate forces into the model that cause policymakers to act in the first place, such as public pressure.

For example, legal theorists who study the regulatory compliance of polluting industries observe that government policy may not be enforced effectively without pressure from citizens (Van Rooij 2010). Social misperception can be the single largest obstacle to resolving pollution problems. Sometimes this is fuelled by an inconclusive scientific understanding, given that the symptoms of pollution exposure can be slow to appear, and difficult to separate from other causes or confounding variables (Van Rooij 2010; Harada 1972; Grandjean et al 2010). Misperception may also be fuelled by economic expediency and deliberate foul play. Therefore, citizen action and changing social perception play a large role in helping to resolve pollution problems.

Recognizing that policy decisions depend on social influences and behaviours that emerge from group interactions, social learning models are increasingly used in mathematical epidemiology to study vaccine scares (Bauch and Bhattacharyya 2012), and in ecology to study resource management and conservation (Satake et al 2007; Innes et al 2013; Barlow et al 2014). The idea is that individuals adopt strategies based partly on their own self-interest, and partly from exchanging information with others in a society to imitate the normative behaviour of the group (Satake et al 2007).

The factory emitted methylmercury directly into Minamata Bay (Jesty 2012; George 2001; Ui 1992; Yorifuji et al 2013), where it posed a hazard to humans and organisms due to its high protein-binding affinity and its potential 
to bioaccumulate rapidly in food chains (Goel 2006; Jackson 1998). As fish was the main source of dietary protein for people in Minamata and was ingested in high quantities (George 2001; Laws 2000; D'Itri 1991), it was not long before people started showing severe neurological symptoms linked with eating fish from the bay. To avoid financial responsibility and pursue record high production, Chisso and its allies in Japan's Ministry of International Trade and Industry concealed test results and stalled independent research that linked the illness to its emissions(Harada 1972; Ui 1992; Smith and Smith 1975).

Faced with ambiguous information and a lack of leadership from the government and industry, the people in Minamata had to resort to their own decision-making and voluntary measures to protect their health. These voluntary actions included fish-eating boycotts and protests to demand pollution abatement. By 1960, fish-eating was reduced, and protests had compelled the government to intervene (Yorifuji et al 2013; Littlefield 1996; Harada 1995; George 2001; Smith and Smith 1975).

Although citizens had acted and secured government attention, the emissions were not completely abated. The pushback from those who supported the company was significant. Many citizens were reticent to blame pollution on a company that they depended upon for their income (Van Rooij 2010), and many viewed the existing pollution victims and the appearance of new victims as a threat to their livelihood. Workers feared that the factory could be shut down (George 2001), and fishermen feared that their fish would not sell (Smith and Smith 1975). This fed a social stigmatization that dissuaded the injured from coming forward with their symptoms, so that the true extent of the damages remained hidden for many years. Believing that the danger was over after 1960 , the community regained confidence in fish-eating even though the pollution continued (George 2001) (Harada 1972). After 1960, nearly 200 people developing the full set of mercury-poisoning symptoms were overlooked, while thousands more were exposed (Harada 1995; George 2001; Harada 1975). This latent pollution epidemic continued until 1968, when a second methylmercurypoisoning incident was discovered in another region of Japan, prompting the government to recognize the pollution problem in Minamata.

Although the Minamata incident occurred 50 years ago, it is very relevant to contemporary pollution problems. Minamata represents highly localized human-environment interactions, and gives us an opportunity to understand both the pollution feedback effects on human health and fishing, and the social feedbacks in response to the pollution.

The HES model that we present in this paper couples phenomena that up to now have been modelled only separately, and introduces new links that allow us to ask new questions. Using differential equations (ODEs), we incorporate social learning into the HES by representing pollution abatement as a dynamic function of the aggregate public demand for it, rather than as a static optimal control parameter. This allows us to examine the role of changing social attitudes, pressures, and misperceptions in the effort to control pollution damages. The demand for abatement changes in response to the changing perception of health risk (Bauch and Bhattacharyya 2012; Innes et al 2013). 
Our objectives are to examine the ecological and social conditions that cause the outbreak of a pollution-induced epidemic, and to study the role of social feedbacks and misperception effects that allow the epidemic to persist.

\section{Methods}

This model couples three subsystems: the population dynamics of a fishery, the dynamics of pollution exposure and the perception of its symptoms, and the social learning dynamics underlying the public pressure to abate pollution. Since parts of this model are developed here for the first time, we provide a detailed model derivation in section S1 of the Supplementary Material.

To maintain our focus on numerical simplicity and qualitative behaviour, we build a system of normalized equations whose state variables are proportions rather than absolute quantities. The system is driven by an exogenous input $n(t)$, which is the baseline emissions loading rate from an industry into a water source. Whereas $n(t)$ is a prescribed function, the net emissions loading $E(t)$ is affected by feedback from the social demand for abatement. The fishery consists of fish $F(t)$ and boats $B(t)$, whose interactions are governed by Lokta-Volterra type equations and emissions mortality.

Human injuries are driven by the fish-borne pollution exposure. This exposure depends on the net emissions level $E(t)$, the fish catch $H F(t) B(t)$ with harvest rate $H$, and a factor of pollution availability to humans, $\epsilon_{I}$.

To model human perception, we introduce $P(t)$ for the perceived injured and $I(t)$ for the actual injured. The parameter $s$ is the stigma rate, or the rate at which the community denies new victims as the perceived injured $P(t)$ increases. This stigma effect causes a disparity between the actual and perceived number of injured. If there is no stigma, $s=0$, then $I(t)$ and $P(t)$ grow at the same rate. However, when $s>0$, then a distinct negative feedback is introduced in $P^{\prime}$ : the rate at which the community is willing to recognize new victims decreases as the number of perceived victims increases. Then $P(t)$ is an underestimate of the actual health damages $I(t)$.

As Figure 1 shows, all social feedbacks and decisions are driven by $P(t)$, but they are blind to $I(t)$. $P(t)$ affects the level of fish-eating, $1-b P(t)$, where $b$ is the boycott rate. $P(t)$ also influences the public demand for abatement $X(t)$, which grows according to a social learning dynamic. In turn, $X(t)$ feeds back to influence the net emissions $E(t)$, thus closing the loop.

Altogether, the equations for the human-environment system are:

$$
\begin{aligned}
F^{\prime}(t) & =r F(t)(1-F(t))-H F(t) B(t)-\epsilon_{F} E(t) F(t) \\
B^{\prime}(t) & =(1-b P(t)) H F(t) B(t)-c B(t) \\
I^{\prime}(t) & =\epsilon_{I} E(t) H F(t) B(t)[1-b P(t)-I(t)] \\
P^{\prime}(t) & =\epsilon_{I} E(t) H F(t) B(t)[1-b P(t)-s P(t)-P(t)] \\
X^{\prime}(t) & =\kappa X(t)(1-X(t))[h P(t)-\beta X(t)]
\end{aligned}
$$

where the net emissions loading $E(t)$ is given by:

$$
E(t)=n(t)(1-f(X(t)))
$$




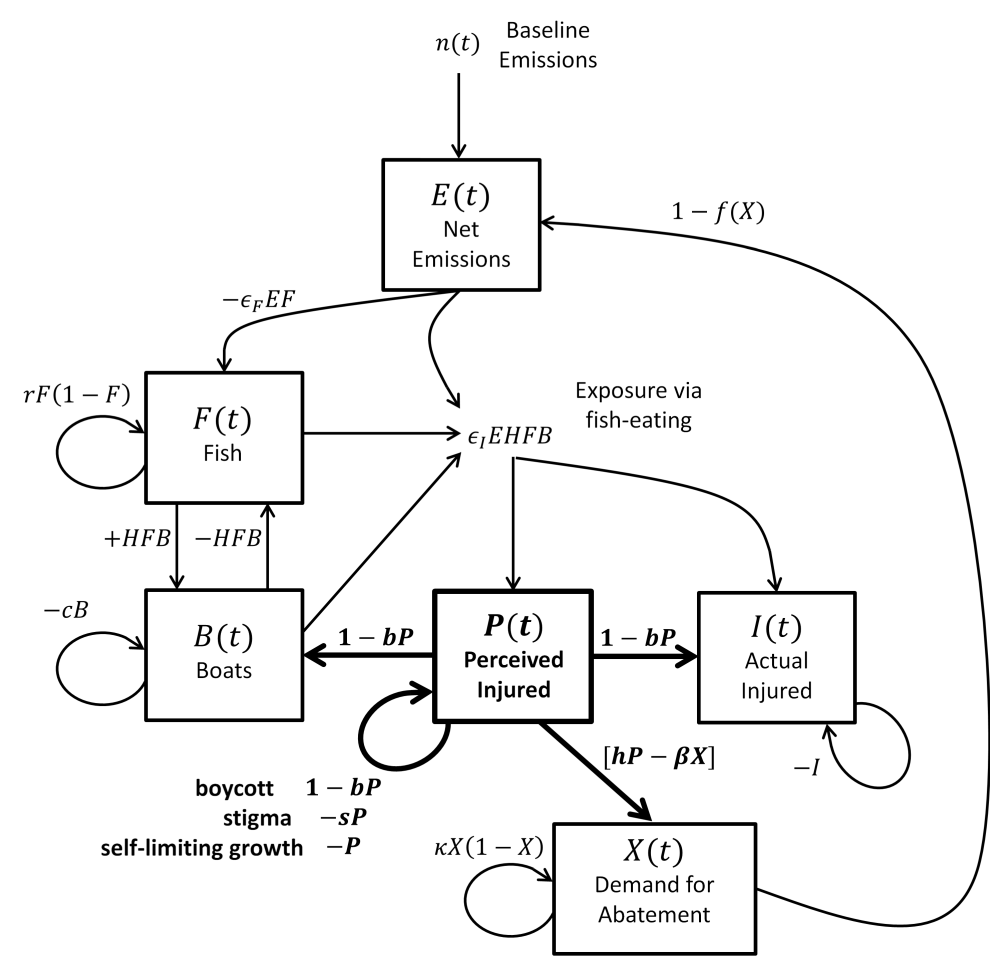

Fig. 1: Causal links and feedback loops among the state variables in the humanenvironment system. Observe the critical role that human perception plays (shown in bold) as a hub for the relay of information. The stigma term $-s P$ acts to dampen the growth of the perceived injured, creating an underestimation of the actual injured. This distorts the signals needed for the fish-eating boycott $-b P$ and the demand for pollution abatement $X$ to fulfil their protective functions.

Note that the form of the abatement function $f(X)$ is important. It should be monotone increasing in $X$, and have a social concern threshold $\breve{x}$ above which there is complete abatement: i.e. whenever $X \geq \breve{x}$, then $f(X)=1$ and $E(t)=0$. Table 1 gives a summary of the model variables and parameters. See the Supplementary Material for more details.

As mentioned in the historical background of Minamata, the emissions may have continued indefinitely (albeit at a reduced, but still dangerous level) and the pollution victims forgotten, if it had not been for a singular event that our model does not capture. That event is the 1965 outbreak of methylmercurypoisoning in Niigata, Japan, at an acetaldehyde factory run by a different company far from Minamata.

Then, in our simulations and analysis, we are interested in the conditions for a nontrivial equilibrium where the emissions and the fish catch coexist (i.e. 
the route of exposure remains open), and where the true number of injured $I$ continues to grow in the background long after the perceived injured $P$ has settled down to its steady-state.

Table 1: HES Model Variables and Parameters

\begin{tabular}{cllc}
\hline Variable & Meaning & Units & Range \\
\hline$E$ & Net Emissions Loading & Proportion & {$[0,1]$} \\
$F$ & Fish & Population proportion & {$[0,1]$} \\
$B$ & Boats & Proportion of $B_{\text {threshold }}$ & $\geq 0$ \\
$I$ & Cumulative Injured & Population proportion & {$[0,1]$} \\
$P$ & Cumulative Perceived Injured & Population proportion & {$[0,1]$} \\
$X$ & Demand for pollution abatement & Population proportion & {$[0,1]$} \\
$f(X)$ & Abatement level & Proportion & {$[0,1]$} \\
\hline \multirow{2}{*}{ Parameter } & Meaning & Units & Range \\
\hline$H$ & Harvesting rate & $1 /$ year & {$[0,1]$} \\
$r$ & Fecundity & $1 /$ year & $(0,1]$ \\
$c$ & Normalized boat costs & $1 /$ year & $(0,1]$ \\
$\epsilon_{F}$ & Fish pollution mortality & $1 /$ year & {$[0,1]$} \\
$\epsilon_{I}$ & Pollution availability to humans & $1 /($ unit emissions $)$ & {$[0,1]$} \\
$b$ & Rate of fish boycott per unit injury & $1 /($ fraction increase in $P)$ & $\geq 0$ \\
$s$ & Rate of stigmatization per unit injury & $1 /($ fraction increase in $P)$ & $\geq 0$ \\
$\kappa$ & Sampling and imitation rate & $1 /$ year & {$[0,1]$} \\
$h$ & Rate of health concern per unit injury & $1 /($ fraction increase in $P)$ & $\geq 0$ \\
$\beta$ & Pushback to demand for abatement & $1 /($ fraction increase in $X)$ & $\geq 0$ \\
$\breve{x}$ & Social concern threshold & Population proportion & {$[0,1]$} \\
\hline
\end{tabular}

Our system has 9 equilibria, with a single nontrivial equilibrium

$$
\left(\begin{array}{l}
F^{*} \\
B^{*} \\
I^{*} \\
P^{*} \\
X^{*}
\end{array}\right)=\left(\begin{array}{l}
\frac{c}{H\left(1-b P^{*}\right)} \\
\frac{1}{H}\left(r\left(1-F^{*}\right)-\epsilon_{F}\left(1-f\left(X^{*}\right)\right)\right) \\
\frac{1+s}{1+b+s} \\
\frac{1}{1+b+s} \\
\frac{h}{\beta(1+b+s)}
\end{array}\right)
$$

In general, this equilibrium exists and is locally stable if the following three inequalities hold. These occur when the social concern is below the threshold needed to abate the emissions,

$$
0 \leq \frac{h}{\beta(1+b+s)}<\breve{x}
$$

when the fish reproduction rate exceeds the pollution mortality,

$$
r>\epsilon_{F}\left(1-f\left(X^{*}\right)\right)
$$

and when the returns from the harvest exceed the cost of fishing,

$$
c<H\left(1-b P^{*}\right)\left(1-\frac{\epsilon_{F}\left(1-f\left(X^{*}\right)\right)}{r}\right) .
$$


However, the HES is sensitive to its initial conditions. Even if the inequalities for a nontrivial equilibrium are satisfied, it will not occur if $F\left(t_{0}\right), B\left(t_{0}\right)$ and $X\left(t_{0}\right)$ are sufficiently small, for a given set of parameters, such that the fish catch collapses before the emissions are reduced. See Supplementary Material sections S2, S3 and S4 for more detail.

\section{Results}

\subsection{Historical Datasets and Parameter Selection}

Most of the data available from Minamata is incomplete or sampled at irregular time intervals, making it ill-suited for a rigorous parameter recovery. Rather than estimate the parameters from the data, we make simulations that agree qualitatively with the information we have from Minamata.

Table 2.1 shows the available datasets, and Table 2.2 summarizes our choice of parameters and initial conditions for the simulation. Table 2.3 contains the simulation plots. Observe that the datasets are reported in absolute numbers, while the simulated variables are plotted as proportions.

To model the baseline emissions $n(t)$, we consider the emissions dataset closely. Prior to 1959, emissions levels increased as part of technical improvements at the factory, the details of which are exogenous to our model. After 1959, emissions were reduced due to public demand for abatement. In our model, we define the following baseline emissions function:

$$
n(t):=\left\{\begin{array}{rcc}
0-100 \% & \text { interpolated data } \quad t \leq 1959 \\
100 \% & \text { (continuing 1959 level) } t>1959
\end{array}\right.
$$

where we take interpolated emissions data up to 1959, and assume that peak levels occur in 1959. The emissions continue at $100 \%$ of 1959 levels if there is no abatement.

To define the relationship between social concern and abatement, we specify a function $f(X)$. Although emissions were abated after 1959, Tsuru reports that many Minamatans supported the company into the 1970s (Tsuru 1999), so it was a vocal minority of citizens that demanded abatement. We take $f$ to be a nonlinear function

$$
f(X):=\left\{\begin{array}{cc}
0 & 0 \leq X \leq 0.2 \\
-250(X-0.2)^{2}(X-0.5) & 0.2 \leq X \leq 0.4 \\
1 & 0.4 \leq X \leq 1
\end{array}\right.
$$

where $\breve{x}=0.4$ is the threshold of social concern above which emissions are fully abated.

There is data for the fish catch in Minamata Bay but not for the actual fish population, so the fish population that we generate in the simulation should be regarded as theoretical. The data for the number of fishing households (1) in Minamata and (2) in the villages Tsukinoura, Detsuki, Yudo, and Modo is used as an indirect estimate for the number of boats in Minamata Bay (Michiko 2003; Tsurumi 2007). 
Table 2: We use historical datasets (Table 2.1) to select parameters for the HES model (Table 2.2) and generate simulations of Minamata (Table 2.3). Sources: emissions data (Harada 1975); fish catch data (Harada 1972); fishing households data (Michiko 2003; Tsurumi 2007); cumulative injured data (Harada 1975); protest events data (Almeida and Stearns 1998).

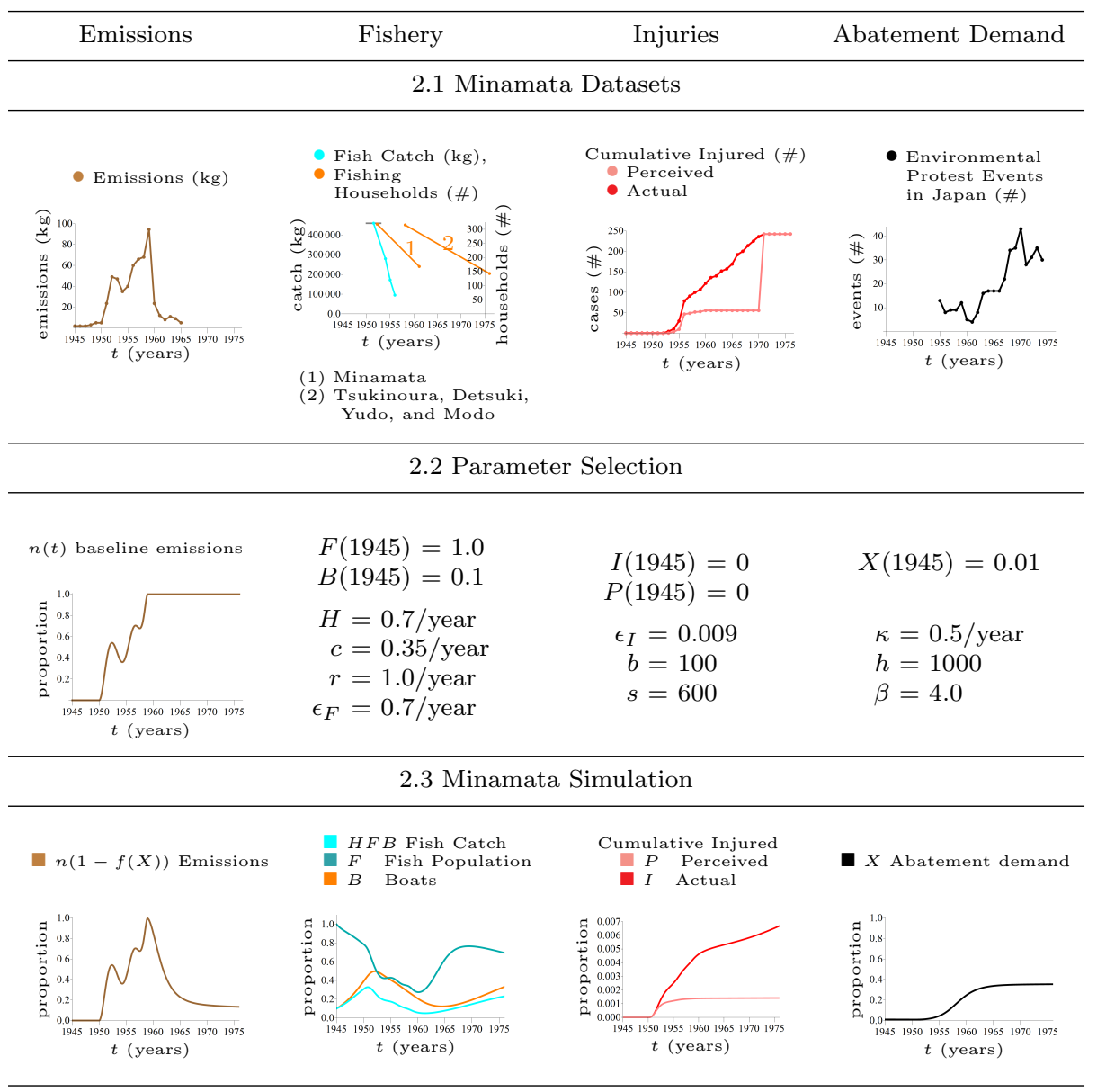

As the fish catch data is incomplete, we also make use of qualitative historical information. In 1945, the fish stocks around Minamata were high, as they had not been fished since before the second world war (Keibo and Masato 2001). After the war, both fishing pressure (Keibo and Masato 2001) and methylmercury pollution reduced the fish stocks. While the data are insufficient to separate the effects of pollution and fish harvesting on the mortality of fish, we can assume that $\epsilon_{F}$ and $H$ together outpace the fecundity $r$. Not shown in the fish catch data, but reported in (George 2001; Harada 1972, 1995) is that after fishing was reduced in 1957 and emissions were reduced in 1959, 
some species were observed to recover to levels deemed fit to fish openly again by 1964 .

The data for the cumulative injured over the years 1945-1976 comes from (Harada 1975). We take the "perceived injured" to be all cases discovered up to 1960 , while the "actual injured" include additional cases discovered retrospectively in a 1971 epidemiological survey. Adjusting for the population size of Minamata over this period, we scale these datasets to estimate $\epsilon_{I}, b$ and $s$. Public opinion poll data about social concern for pollution is not available from Minamata. Instead, we observe that social concern toward pollution at the nationwide-level in Japan underwent an increase during this period, judging from annual time series data on the number of anti-pollution social movement activities reported in Japanese newspapers, journals and magazines from 1955-1974 (Almeida and Stearns 1998). These activities include marches, rallies, demonstrations, and demands made by anti-pollution groups.

\subsection{Simulations and Scenario Analysis}

Given that the social parameters $s, b$ and $h$ have been selected to agree qualitatively with the data, we concede that there is substantial uncertainty in the values selected. To provide context for the choices made in Table 2.2, we depict the steady-states of the system in $s$ - $b$ parameter space. We numerically solve $F, B$ and $X$ for large time $t=1000000$, and colour regions in $s-b$ space according to the equilibrium type (I, II, III or IV) as defined in Table 3. See Supplementary Material sections S3 and S4 for more detail.

Table 3: Equilibria Types (and associated Region Colours)

\begin{tabular}{|c|c|c|}
\hline & $1-f\left(X^{*}\right)=0$ & $1-f\left(X^{*}\right)>0$ \\
\hline$F^{*} B^{*}=0$ & $\begin{array}{l}\text { I) No Fish Catch, } \\
\text { No Emissions }\end{array}$ & $\begin{array}{l}\text { III) No Fish Catch, } \\
\text { Emissions }\end{array}$ \\
\hline$F^{*} B^{*}>0$ & $\begin{array}{l}\text { II) Fish Catch, } \\
\text { No Emissions }\end{array}$ & $\begin{array}{l}\text { IV) Fish Catch, } \\
\text { and Emissions }\end{array}$ \\
\hline
\end{tabular}

The coloured $s-b$ space is shown in Figure 2.c, and its regions are divided by numbered and coloured contour lines, given in Legend 1. These contour lines are based on the inequalities (4)-(6), as explained in more detail in section S5 of the Supplementary Material.

The $s-b$ space in Figure 2.c is accompanied by representative time series plots in Figures 2.a,b,d,e. Each figure depicts solutions to the HES system that illustrate the dynamic behaviour of a particular equilibrium type (I, II, III, or IV). The time series in these plots are coloured according to Legend 2 . 

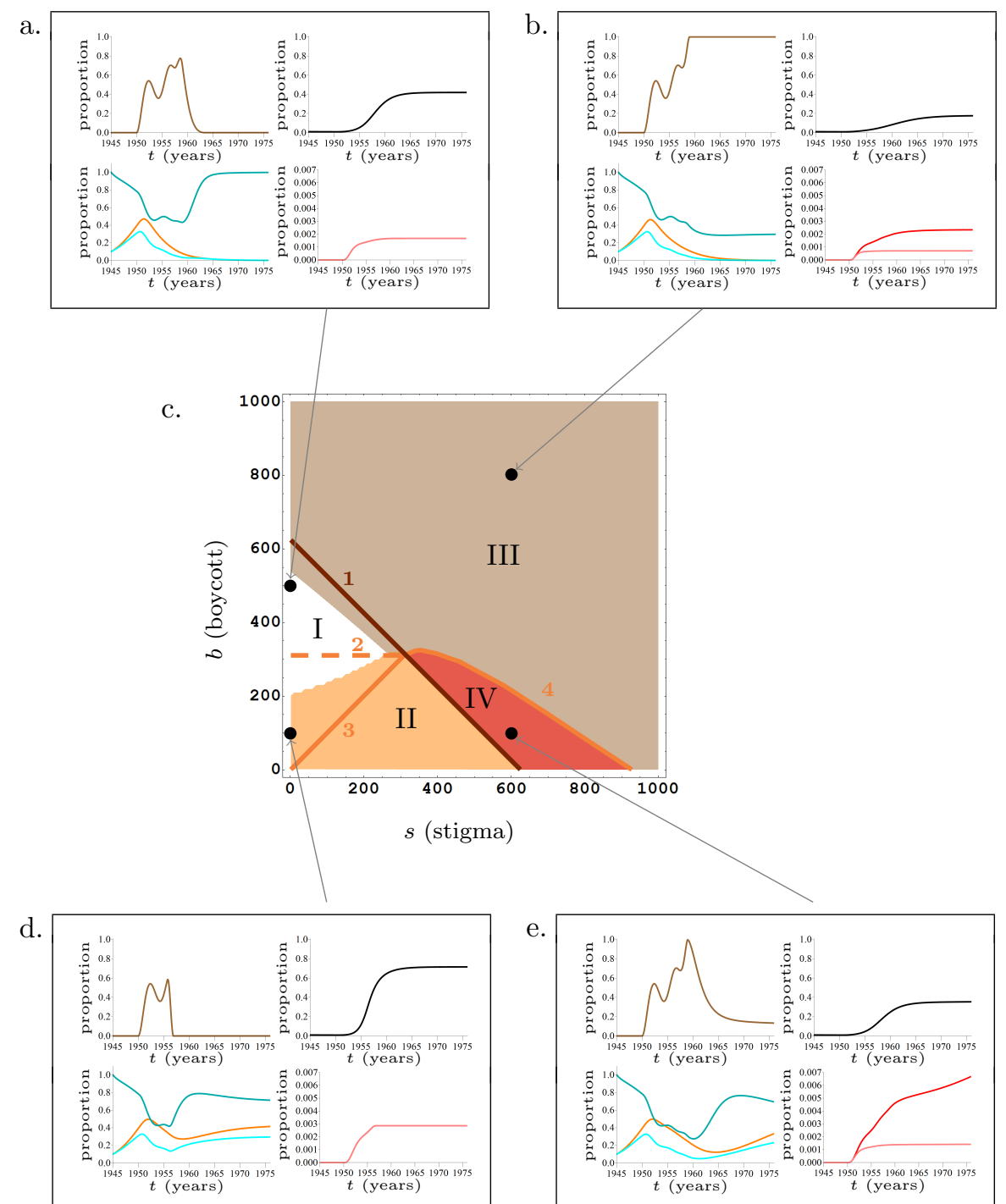

Fig. 2: Changing values for the social feedbacks $s$ (stigma) and $b$ (boycott) yield alternative qualitative outcomes. The parameter plane (c) shows the dynamical outcomes defined by the equilibrium-types I, II, III, and IV that result for various values of $s$ and $b$. The accompanying time series plots are simulated at fixed values of $s$ and $b$, and each is representative of a particular outcome: (a) I: $s=0, b=500$, (b) III: $s=600, b=800$, (d) I: $s=0, b=100$, (e) IV: $s=600, b=100$. The scenario in (e) corresponds to the Minamata simulation defined in Table 2.3. 


\subsubsection{Stigma/Misperception Undermines Protective Social Feedbacks}

Figure 2.e depicts the Minamata simulation that we generated in the previous section using $s=600, b=100$. Consider our simulation compared to the other scenarios, in the context of changing stigma $s$ and boycott pressure $b$.

Figure 2.a shows a type-I (No Emissions, No Fish Catch) scenario for $s=$ $0, b=500$. Increasing the boycott rate $b$ reduces the number of people exposed to the pollution. However it also lowers the demand for fish, which increases the cost to harvest ratio. For $b$ sufficiently large the fish catch is no longer viable.

Figure 2.d shows a type-II (Fish Catch, No Emissions) scenario for $s=$ $0, b=100$. We find that for $s$ and $b$ sufficiently low, enough people are exposed to the pollution that social concern rises and the emissions are eliminated. The boycott rate $b$ is sufficiently low that the fish catch survives.

Figure 2.b shows a type-III (Emissions, No Fish Catch) scenario for $s=$ $600, b=800$. This scenario occurs when either the stigma or boycott pressure are sufficiently high. If the boycott rate $b$ is increased, then people stop eating fish to protect themselves. If they boycott at such a rate that the fish catch collapses before enough people have been injured to cause full abatement, then the emissions survive. On the other hand, the stigma $s$ has no preventative effect to truly protect people from injury. Increasing stigma causes an underestimation of the true injuries. If the stigma $s$ is increased, or if $s$ and $b$ are both made sufficiently large, then the number of perceived injured is too low to stimulate emissions abatement. In addition, if $s$ is high enough then the abatement is low, and the fish catch loses its viability due to pollution.

The Minamata scenario results when the boycott rate $b$ is low enough to allow the fish catch to survive, and the stigma rate $s$ is high enough that injuries are underestimated and a pollution epidemic occurs.

\subsubsection{Social Trade-offs}

To reinforce what the time series plots in Figures 2.a,b,d,e show us about the effect of varying boycott and stigma on the cumulative injuries, we approximate $I^{*}$ by numerically solving $I$ for large time $t=1000000$, over a $1000 \times 1000$ grid of $s$ and $b$, and plot the distribution of injuries in $s$ - $b$ space. Figure 3 presents this plot next to a coloured region plot in $s-b$ space that demarcates the various equilibrium types I, II, III and IV.

We find that injuries are by far the highest in region type-IV. In general, injuries are higher with increasing stigma, and lower with increasing boycott pressure. With low stigma and high boycott pressure, there is a social tradeoff: a high number of injuries are prevented, but this happens at the expense of the fishery, which collapses.

Observe that the simulations which result in the least number of cumulative injured come with collateral disadvantages: steady-states I and III result in the collapse of the fishery, which is a collapse of the economic livelihood of a people. Steady-state III allows the emissions to continue unabated, and by 
comparing Figures 2.a,b,d,e, one can see the effect this has on stunting the fish population. This would have major ecological ramifications for the wider food web, which are not modelled here.

a.

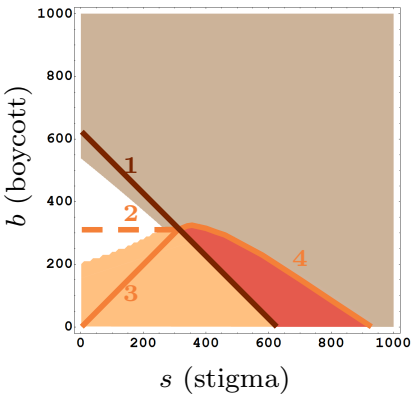

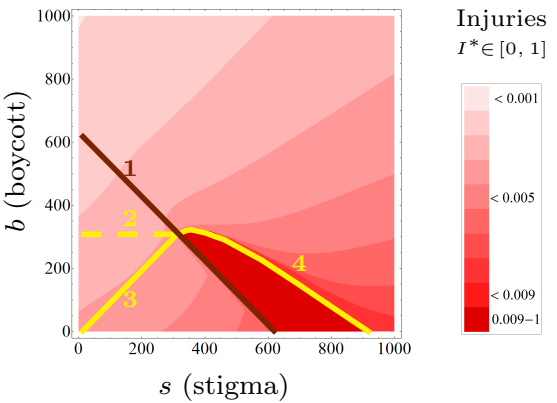

Fig. 3: Parameter planes showing outcomes for various values of $s$ and $b$. Plots show (a) the dynamical outcomes defined by the equilibrium-types I, II, III, and IV, and (b) health outcomes defined by gradations in the cumulative injured steady state, $I^{*} \in[0,1]$.

\subsubsection{Sensitivity to Initial Conditions}

As discussed in Section 2, the qualitative behaviour of the system depends on the initial conditions for the fishery. If $F\left(t_{0}\right) B\left(t_{0}\right)$ is sufficiently small, then $F B \rightarrow 0$ faster than either $P \rightarrow P^{*}$ or $f(X) \rightarrow 1$. In regions where we expect a type I, II or IV equilibrium, the fish catch collapses before emissions are abated and we get a type-III equilibrium. This is shown in Figure 4.
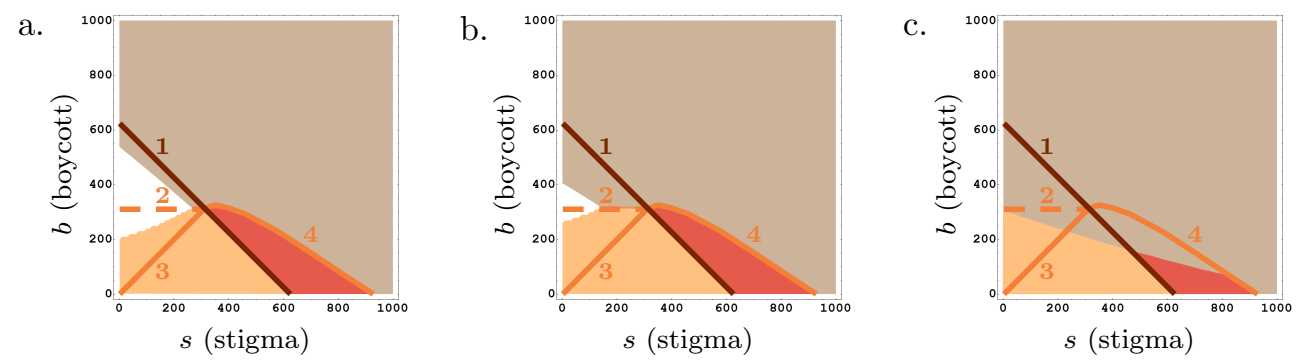

Fig. 4: Parameter planes showing dynamical outcomes I, II, III, and IV for various values of $s$ and $b$ with different initial fish population densities: (a) $F(1945)=1$, (b) $F(1945)=0.1$, (c) $F(1945)=0.01$. This demonstrates the system's dependence on its initial conditions. 


\subsubsection{A Pollution Epidemic is Unlikely Without Stigma/Misperception}

It is very important to recognize how unlikely the conditions for the pollution epidemic (or type-IV equilibrium) are without social stigma/misperception. As Figure 2 shows us, the type-IV equilibrium does not exist unless $s>0$ and $s$ is sufficiently large.

We ask: what parameters are required for us to have a pollution epidemic when $s=0$, and how physically plausible are they compared to the parameters in Table 2.2 that we used in our simulation?

First let us consider decreasing $h$, the health concern, so that region IV intersects with the $b$-axis. Theoretically, the largest value of $h$ needed for this to occur is when $s=0$ and the contour lines $\frac{h}{\beta(1+b)}=0.4$ and $c<$ $H\left(1-b\left(\frac{1}{1+b}\right)\right)$ intersect. Assuming that the injuries grow to $P^{*}=\frac{1}{1+b+s}$ and the demand for abatement is $X^{*}=\frac{h}{\beta} \frac{1}{1+b+s}$, this intersection should occur when $h=3.2$

a.

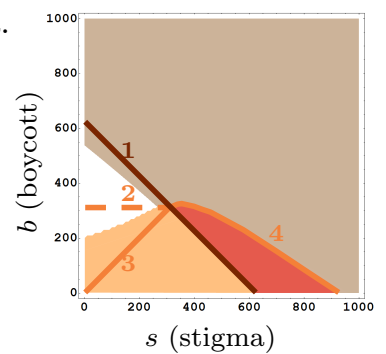

c.

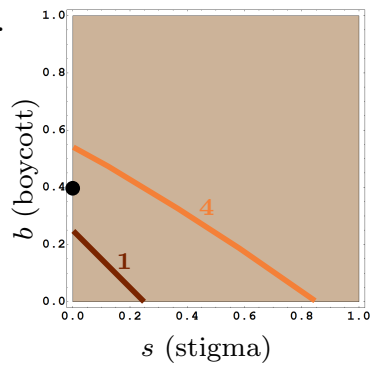

b.

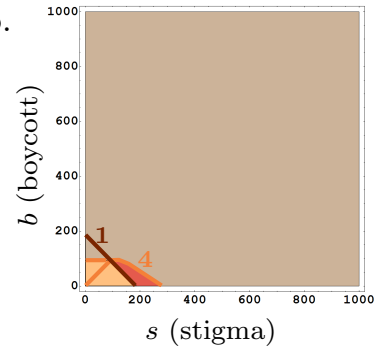

d.

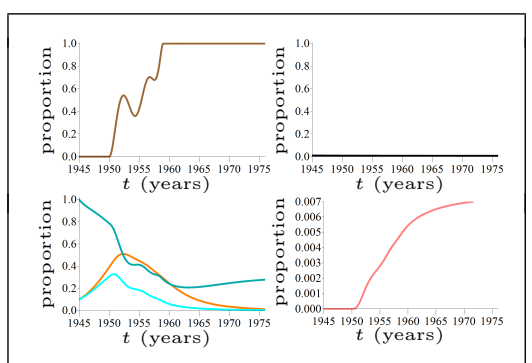

Fig. 5: Parameter planes showing dynamical regimes for various values of $b$ and $s$, with decreasing health concern: (a) $h=1000$, (b) $h=300$, (c) $h=3$. The time series plots in (d) show solutions of the system for $h=3, s=0$, $b=0.4$. We find that the small values of $h$ and $b$ make the social response to injuries negligible. Emissions are unabated and the fish catch collapses. An ongoing pollution epidemic is averted. 
However, as Figure 5 shows, if we solve the steady-states numerically for $h=3, s=0$ and $b=0.4$, we find that a pollution epidemic does not occur in the region where we expect it to. In effect, the very small $h$ and $b$ make the social repsonse to the pollution negligible. The fish catch collapses before the injuries grow large enough to trigger any abatement. Emissions continue but exposure stops, so a pollution epidemic is averted.

In the absence of misperception, a pollution epidemic is possible provided that the social feedbacks to stop it are negligible and the fish catch survives. However, we find that it is unlikely for the fish catch to coexist with unabated emissions.

In the case of Minamata, the social feedbacks were not neglible; the fish boycott and demand for abatement were significant feedbacks. Unfortunately, the reduction in emissions made it possible for the fish catch to coexist with the emissions.

a.

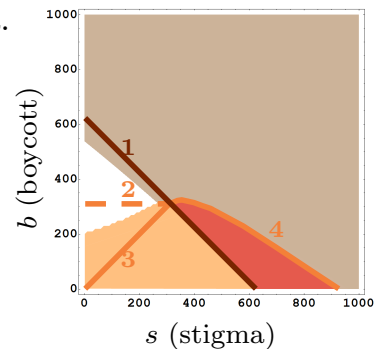

c.

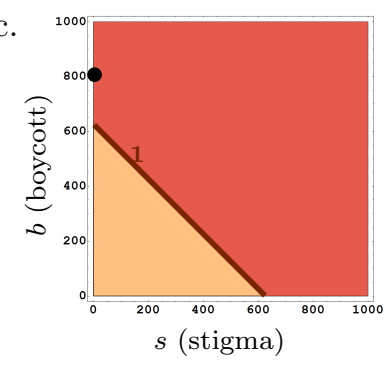

b.

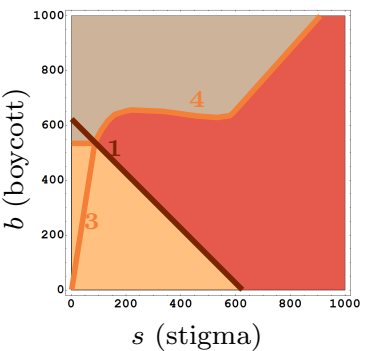

d.

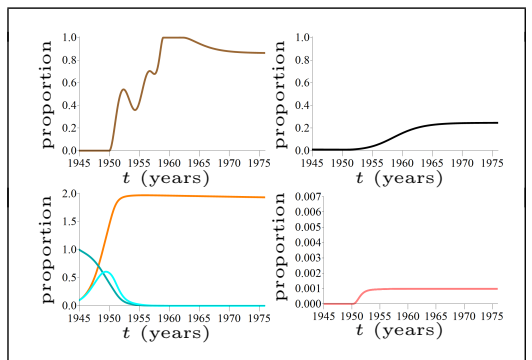

Fig. 6: Parameter planes showing dynamical regimes for various values of $b$ and $s$, with decreasing boat costs: (a) $c=0.35$, (b) $c=0.1$, (c) $c=0.001$. The time series plots in (d) show solutions of the system for $c=0.001, s=0, b=800$. We find that the boats survive at an unrealistically high level. The growth of the boats forces the fish population toward zero. Over a short time-scale, $I^{\prime} \approx 0$ and growth of injuries is negligible. 
Now let us consider changing the boat costs. With $c<0.0012$, we find that region IV intersects the $b$-axis. As the numerical simulations in Figure 6 show, the operating cost for the boats is unrealistically low, the boats grow to an unrealistically high level and push the fish population toward zero. For the short time-scale we are interested in, the potential for a pollution epidemic is averted by the decline of the fish catch to near-zero levels. If we solve numerically as $t \rightarrow \infty$, it turns out that the fish and boats exhibit damped oscillations, and the injuries grow.

Then, in the absence of misperception, we do find that a pollution epidemic is possible with reduced boat costs, but that it relies on the unlikely and infeasible condition that a large number of boats survives by fishing a nearzero fish population over a large time scale.

Therefore, a pollution epidemic relies on the presence of stigma or misperception.

\section{Discussion}

The coupled HES model presented in this paper allows us to investigate the dynamics of pollution exposure as intimately linked to the local environment, the economy, and social decisions. The analysis and simulations of this model show us at least three important things: (1) increasing stigma/misperception undermines the social feedbacks that protect people from pollution. (2) A pollution epidemic steady-state occurs when emissions and fish ingestion continue indefinitely. The occurrence of this steady-state is highly unlikely without stigma/misperception. (3) With multiple socially desirable outcomes, feedbacks can cause one outcome to undermine the other. Here, the scenarios that prevent the most injuries also cause the commercial fishery to collapse.

This model represents misperception effects in a novel and critical way. In the model, social decisions to boycott fish-eating and abate pollution are made from information about the perceived variables, $F, B, P$ and $X$. These variables can reach a false equilibrium that is blind to $I$, the actual injuries, which grow unperceived in the background. The prevention of injuries involves a trade-off: either the emissions or the fish catch must stop. One is a cost to the polluting industry, the other is a cost to the fishing industry.

In the context of the Minamata pollution epidemic, this HES model represents the conditions that might have persisted for decades in Minamata if a second methylmercury-poisoning incident had not occurred in Niigata, Japan. This model is focused on the localized relationships that are endogenous to Minamata and its people, which allowed the social response to pollution to settle to a false equilibrium. It remains future work to incorporate the effects of a singular external event such as Niigata that shook Minamata from its false equilibrium.

In the case of Minamata, the neglect of the government and industry fed a stigma of Minamata's pollution victims. Although the extent of the neglect and the stigma may seem like relics of a different time and place, they are not. For 
example, the recent struggle of residents in Mossville Louisiana (Subra 2007; Livingston 2013), and the ongoing struggles of activists in China's cancer villages (Tianjie 2009; Van Rooij 2010) show that the pursuit of economic expediency at the expense of human welfare remains a pivotal obstacle in resolving pollution epidemics.

The model has several limitations that are common to simple compartmental ODE models, and others that are particular to the phenomenon being modelled, such as social dynamics and dose-response relationships. Overall, the modeller must find a balance between detailed realism on one hand, and a parsimonious representation that is mathematically tractable, on the other.

Our model takes the pollution to be spatially localized and homogeneously distributed in a closed bay. This neglects the role of diffusion and fish migration in spreading the contamination to people beyond Minamata bay. We also assume that the social dynamics are independent of space. This is unrealistic insofar as social concern and demand for abatement is stronger among population groups that live farther from the polluting industry.

The model aggregates individual and species-level variability. The use of a single variable $F$ for all sea animals obscures the multi-species interactions and diversity of a real ecosystem. The assumption that the pollutant concentration in fish is population-averaged neglects variability due to fish size, age and location. Similarly, by modelling the human exposure to pollution as population-averaged, we unrealistically assume that all fish-eaters ingest at the same rate and face the same risks. We class all human injuries as "injured", regardless of the severity or type of symptoms. These aggregations can be corrected by adding population compartments for species, age, or fish-eating classes to our equations. Alternatively, the system can be hybridized with a discrete agent-based model that represents individual-level variability using a network of interacting agents.

Another assumption is that the accumulation of the pollutant in fish is directly proportional to the emissions loading at any time, without delay. This circumvents the use of mass-balance equations to model the accumulation process of the pollutant in water, sediment, and organisms. In the model, fish mortality and human injury occur upon contact with the pollutant, without delay. Although Methylmercury does rapidly accumulate and cause damage to organisms, when it is encountered chronically and at lower concentrations, a time lag becomes relevant. We can introduce mass-balance equations to represent more steps in the pollutant accumulation, or introduce time delay terms to the existing equations.

Note that the model takes pollution injuries to be irreversible. This is a reasonable assumption for methylmercury-poisoning, where the severity of some symptoms may be reduced by medical treatment but most patients remain injured for life. Our model is not suitable to model pollution sicknesses that can be treated quickly.

We also remark that the utility function in the equation for $X^{\prime}$ could be improved by including a term to represent the fishermens' concern over damage to the fish catch. 
Lastly, we observe that the model represents a localized social learning process which is isolated from external events, and external opinions. The Niigata pollution epidemic was an event that changed the social attitudes of people across Japan to oppose the polluting industry in Minamata. Concerned citizens in Minamata depended on external allies and protests to galvanize public attention for their cause. To model a singular external effect such as Niigata, we can introduce an impulse term $+\eta(t)(I-P)$ in the equation for $P^{\prime}(\mathrm{t})$, which acts to correct misperception given an impulse $\eta(t)>0$.

Despite these limitations, coupled HES models can provide insights regarding the interactions between natural dynamics, pollution dynamics, and human social dynamics. Recent cases from Mossville, Lousiana, and from China's "cancer villages" emphasize the relevance of stigma and misperception to contemporary pollution problems. Coupled HES models that include misperception effects as part of their human-environment interactions are highly topical and should be further studied.

Acknowledgements We give special thanks to Dr. Akiko Satake and her student Keita Honjo for their stimulating discussions. The first author thanks the Ontario Graduate Scholarship program for financial support, and the second author thanks the Natural Sciences and Engineering Research Council of Canada.

\section{Figure Legends}

$$
\begin{aligned}
& \text { Legend 1: } \\
& \text { Contour Line Colours } \\
& \begin{array}{l}
\text { 1) } h=0.4 \beta(1+b+s) \\
\text { 2) } c=H\left(1-b\left(\frac{0.4 \beta}{h}\right)\right) \\
\text { 3) } c=H\left(1-b\left(\frac{1}{1+b+s}\right)\right) \\
\text { 4) } c=H\left(1-b\left(\frac{1}{1+b+s}\right)\right) \\
\quad\left(1-\frac{\epsilon_{F}}{r}\left(1-f\left(\frac{h}{\beta(1+b+s)}\right)\right)\right) \\
\text { 5) } r=\epsilon_{F}\left(1-\frac{h}{\beta(1+b+s)}\right)
\end{array}
\end{aligned}
$$

Legend 2:

Time Series Plot Colours

$n(t)(1-f(X))$
$X$
$H F B$
$F$
$B$
$P$
$I$

\section{References}

Almeida P, Stearns LB (1998) Political Opportunities and Local Grassroots Environmental Movements: The Case of Minamata. Social Problems 45(1):37-60

Anderies JM (2014) Understanding the Dynamics of Sustainable SocialEcological Systems: Human Behavior, Institutions, and Regulatory Feedback Networks. Bulletin of Mathematical Biology Epub

Barlow LA, Cecile J, Bauch CT, Anand M (2014) Modelling Interactions between Forest Pest Invasions and Human Decisions Regarding Firewood Transport Restrictions. PLoS ONE 9(4) 
Bauch CT, Bhattacharyya S (2012) Evolutionary Game Theory and Social Learning Can Determine How Vaccine Scares Unfold. PLoS Computational Biology 8(4):1-12

D'Itri FM (1991) Mercury Contamination - What We Have Learned Since Minamata. In: Lee HK (ed) Fourth Symposium on our Environment, Springer Netherlands, pp 165-182

Dubey B (2010) A Model for the Effect of Pollution on a Human Population Dependent on a Resource with Environmental and Health Policy. Journal of Biological Systems 18(3):571-592

Eichner T, Pethig R (2006) Economic Land Use, Ecosystem Services and Microfounded Species Dynamics. Journal of Environmental Economics and Management 52(3):707-720

George TS (2001) Minamata: Pollution and the Struggle for Democracy in Postwar Japan. No. 194 in Harvard East Asian Monographs, Harvard University Asia Center, Cambridge Mass. and London

Goel P (2006) Chapter 13.7: Heavy Metal Pollution - Mercury. In: Water Pollution: Causes, Effects and Control, New Age International, New Delhi, pp $147-150$

Grandjean P, et al (2010) Adverse Effects of Methylmercury: Environmental Health Research Implications. Environmental Health Perspectives 118(8):1137-1145

Harada M (1972) Sachie, T. and T. S. George (English Trans.) (2004). Minamata Disease. Minamata Disease Patients Alliance, Tokyo

Harada M (1975) Smith A. (English Trans.). Minamta Disease: A Medical Report. In: Smith E, Smith A (eds) Minamata, Holt, Rinehart and Winston, New York, pp 180-192

Harada M (1995) Minamata Disease: Methylmercury Poisoning in Japan Caused by Environmental Pollution. Critical Reviews in Toxicology 25(1):124

Heijnen P, Wagener F (2013) Avoiding an Ecological Regime Shift is Sound Economic Policy. Journal of Economic Dynamics and Control 37:1322-1341

Innes C, et al (2013) The Impact of Human-Environmental Interactions on the Stability of Forest-grassland Mosaic Ecosystems. Nature, Scientific Reports 3

Jackson TA (1998) Chapter 5: Mercury in aquatic ecosystems. In: Langston W, Bebianno M (eds) Metal Metabolism in Aquatic Environments, Ecotoxicology Series 7, Chapman \& Hall, London, pp 77-158

Jesty J (2012) Chapter 8: Making Mercury Visible: the Minamata Documentaries of Tsuchimoto Noriaki. In: Zuber SL, Newman MC (eds) Mercury Pollution: A Transdisciplinary Treatment, CRC Press, Taylor \& Francis Group, pp 139-160

Keibo O, Masato O (2001) Colligan-Taylor, K. (English Trans.) Rowing the Eternal Sea: the Story of a Minamata Fisherman. Rowman \& Littlefield Publishers, Inc., Lanham/Boulder/New York/Oxford

Laws EA (2000) Chapter 12.3.d: Minamata Disease. In: Aquatic Pollution: An Introductory Text, John Wiley \& Sons, New York, pp 389-397 
Levin S, Clark WC (2010) Toward a science of sustainability. In: Center for International Development Working Papers 196, John F. Kennedy School of Government, Harvard University

Littlefield A (1996) 246: Minamata Bay Pollution in Japan and Health Impacts. Trade \& Environment Database (TED) Case Studies 5(1), http://www1.american.edu/TED/mimamata.htm

Liu J, et al (2007) Coupled Human and Natural Systems. AMBIO: A Journal of the Human Environment - BioOne 36(8):639-649

Livingston JK (2013) Mossville crisis goes to international court. Louisiana Weekly, 22 April 2013. Available: http://www.louisianaweekly.com/mossville-crisis-goes-to-internationalcourt/ [Last accessed: 16 June 2014]

Michiko I (2003) Monnet, L. (English Trans.) Paradise in the Sea of Sorrow: Our Minamata Disease. Center for Japanese Studies, The University of Michigan, Ann Arbor

Satake A, et al (2007) Synchronized Deforestation Induced by Social Learning Under Uncertainty of Forest-use Value. Ecological Economics 63(2):452-462

Smith E, Smith A (1975) Minamata. Holt, Rinehart and Winston, New York

Subra W (2007) Industrial Sources of Dioxin Poisoning in Mossville, Louisiana - A Report Based on the Government's Own Data. Mossville Environmental Action Now (M.E.A.N) Inc., The Subra Company, Advocates for Environmental Human Rights (AEHR), New Orleans and Washington DC

Tianjie M (2009) Environmental Mass Incidents in Rural China: Examining Large-Scale Unrest in Dongyang, Zhejiang. Issue 10 in series China Environment Series, Woodrow Wilson International Center for Scholars, Washington DC

Tsuru S (1999) Chapter 4: Historical Analysis - the Postwar Period ii: Major Kogai Incidents. In: The Political Economy of the Environment: The Case of Japan, UBC Press, Vancouver, pp 70-115

Tsurumi Y (2007) Minamata's Moyainaoshi Movement and Sustainable Development: Recovery from Division. In: Education for Sustainable Development, The ESD Study Group for the Asia-Pacific Region, Tokyo

Ui J (1992) Chapter 4: Minamata Disease. In: Ui J (ed) Industrial pollution in Japan, United Nations University Press, University of Okinawa, Tokyo, pp $103-132$

Van Rooij B (2010) The People vs. Pollution: Understanding Citizen Action Against Pollution in China. Journal of Contemporary China 19(63):56-77

Withagen C (1995) Pollution, Abatement and Balanced Growth. Environmental and Resource Economics 5:1-8

Yorifuji T, Tsuda T, Harada M (2013) Chapter 5: Minamata disease: a challenge for democracy and justice. In: Part A - Lessons from health hazards, in series Late Lessons from Early Warnings: Science, Precaution, Innovation, EEA (European Environment Agency), Copenhagen, pp 124-162 